Department of Public Health, Epidemiology and Biostatistics University of Birmingham

Edgbaston

Birmingham B15 2TT, UK

\section{Can a health professional represent patient views: HTA response}

doi:10.1017/S0266462311000420

\section{To the Editor:}

As a health technology assessment body committed to active patient and public involvement, we at the Scottish Medicines Consortium (SMC) have considered the question above and the views expressed by Dr. Claire Packer and conclude that the answer is "Yes." As Dr. Packer notes, no patient representative can ever be purely a patient with a specific condition as everyone brings other life experiences, including but not limited to their employment, to their understanding, and perception of their condition. A background in health care will provide different experiences from other backgrounds, but these experiences bring added breadth to the patient perception and, thus, enhance any health technology assessment or other decision making in the health arena.

Clearly a patient with a background in finance, education, or manufacturing industry would bring different experiences, but these are no more (or less) valid or valuable than the experiences of a healthcare professional. Importantly, patient representatives in healthcare decision making have a role to represent as wide a range of patients as possible and not simply themselves. This is a difficult challenge and one which no organization has fully resolved, but there is no reason to expect that a patient with a healthcare background would be any less able to fulfill this role than any other patient. Involvement of several patients from different backgrounds is ideal, and where established Patient Interest Groups already exist, their involvement (subject to appropriate declarations of interest) can be very helpful and avoid over-reliance on individual patients. No individual sub-group of patients should be systematically excluded from the process.

As Dr. Packer notes, healthcare professionals experience the same range of emotional and psychological responses to the development of both acute and chronic illness as other patients, and may struggle to find the support and help that they need. To suggest that their experiences have no relevance or value in healthcare decision making is entirely inappropriate.

\section{Ken Paterson}

Email: ken.paterson@dsl.pipex.com

Chair, Scottish Medicines Consortium

\section{Delta House}

50 West Nile Street

Glasgow G1 2NP

Scotland

\section{Can a health professional represent patient view: Patient organization response}

doi:10.1017/S0266462311000432

To the Editor:

A healthcare professional expressed anger and dismay in response to a comment reportedly made by a patient group representative that "health professionals (even those who are also patients) were not considered as representatives for patient groups in decision-making fora." I have heard this sentiment before and always believed it begs the question, "What qualifies one to be a patient representative, and are there any disqualifiers?"

Obviously, not just anyone who has a health condition could appropriately represent the "patient perspective" in a decision-making context. He/she would need to know the views of the patients affected by the decision and also be able to represent these views in a multi-stakeholder forum. $\mathrm{He} / \mathrm{she}$ potentially could be a board member or staff of a patient organization.

Presumably, in a multi-stakeholder forum, the patient representative would advocate for the best interests of the "recipients" of healthcare in the same way as others advocate for the interests of the "regulator," "public payer," the "healthcare institution," and the "healthcare provider." Could the reviewer, the bureaucrat, the administrator, or the health professional also be a patient? Based on the statistics, at least half of them are. However, they participate in their professional roles, not their personal roles.

The representative for the patient presumably also has other roles in life, but he/she will be speaking in the role of patient. He/she could be a teacher, a chef, a housekeeper, bank executive, lawyer, politician, and, yes, even a dentist, pharmacist, nurse, or doctor.

There are obviously many advantages to having patient representatives at the decision table who can easily understand the perspectives of the other stakeholders and who are respected by the others. There are, however, two key challenges for the patient representative who also has (or had) one of the other stakeholder roles. Can he/she manage the potential conflicts of interest and strongly advocate as a patient? More importantly, can he/she maintain the trust of the patients represented? In reality, these are personal qualifications for any patient advocate, and not all patients have them. The health professional chosen to represent patients just has to work a little bit harder to demonstrate them.

Durhane Wong-Rieger, $\mathrm{PhD}$

Email:durhane@sympatico.ca

Chair-Elect, International Alliance of Patients'

Organizations

703 The Chandery 
50 Westminster Bridge Road

London SE1 7QY, UK

\section{Can a health professional represent patient views: Industry response}

doi:10.1017/S0266462311000390

To the Editor:

The writer raises some interesting points. It is evident the "health professional as patient" faces several challenges that are of a general nature and not specific to Health Technology Assessment processes. As she articulates, the "health professional as patient" may be faced with a range of responses including the assumption that the "patient" knows everything relevant to their condition and does not need support, or somehow feeling the health professional has "let the side down" by becoming a patient.

In regard to HTA processes specifically, there is increasing recognition of the importance of quality, balanced input from patients, patient advocates, and in some situations, carers. The fact that the health professional concerned may, by virtue of professional knowledge and experience, have an advantage in expressing that input should not be seen as a reason for preventing or ignoring that input. On the contrary, provided the HTA process seeks and uses patient (and carer) perspectives for the right reasons, this should be a positive. The "right reasons" include gaining a more thorough understanding of what it is like to live with the condition in question, clarifying what changes in disease-specific outcome measures really mean, and the strengths and disadvantages of current treatments.

Conducted with these objectives in mind, patient and public perspectives can be a positive contribution to good HTA. There is no place for the "this treatment must be recommended because I need it" style of input. Indeed, that can be seen on occasions from health professionals and patients alike. What is needed is a positive and supportive HTA process that makes clear what input is desired from both patients and health professionals, and how that will be used. When this is operating and individuals understand what is useful, they should feel that their input is both welcome and valuable to the HTA process and its outputs.

From an industry perspective, our desire is to see the full range of perspectives considered in HTA processes, with objective assessment that considers all stakeholders views and inputs. This requires a transparent system and education for all stakeholders, including patient advocates and interested health professionals.

David Grainger, BS

Email: grainger_david@lilly.com

Global Public Policy Director
Eli Lilly and Company

Lilly Corporate Center

Mail Drop Code 1852

Indianapolis, Indiana 46285

\section{Response: I know how you think, so I can help}

doi:10.1017/S0266462311000407

\section{To the Editor:}

I do think all of us are clear that (i) we don't want to be sick, and (ii) being a patient is a matter of time, so almost anybody could walk away from the disease journey. I do really subscribe to both statements, although, unfortunately, I am living with an incurable and rare form of malignant tumor, aka cancer, since the year 2001. Moreover, I have been on daily chemotherapy for the past 4 years and have been exposed over time to three major surgeries and six different types of chemotherapy.

Despite cancer, recently, I have been quite busy on learning how to manage an intensive insulin therapy needed to live with postsurgical diabetes. All these maladies have been compatible to more than 500 lectures given in the past 3 years in different parts of the world, sitting in more than 30 government and nongovernment positions, having a CEO position in a $\$ 2 \mathrm{M}$ annual budget Foundation for 12 years, creating several projects, enjoying a marriage of more than 20 years which includes two wonderful full-of-life boys, and writing over 200 papers of all kinds, including three nonfiction books and a novel. Am I a kind of superman? No way, I am a cancer patient! I know that life is short and is a gift that deserves to be lived intensively and with joy. Let others have the problems; let's focus on the solutions.

I don't care if I have done much or little, but I care about fulfilling a moral obligation to share my painful experience with others because what is happening to me might happen to you anytime. And, if that thing happens to you, I can assure you that you'd like to benefit from all helpful experiences at hand. Mine is one of them. However, my moral obligation to share personal experience is grounded on three major factors. First, it fits with my aim of preserving human dignity in front of the stigma surrounding cancer patients. Nobody wants to talk about it, but the stigma is always there. Second, it fits with my professional sense of purpose. I chose to be a doctor because I wanted to serve people facing vulnerability. Third, I have received from society more than I deserve and more than I could give back. I have an almost free medical care, including the latest innovations; an almost free medical school, a free 4 years of doctoral studies at Harvard, an almost free $\mathrm{PhD}$ in sociology, an interesting experience in government, etc. Despite an unfair and painful disease, I have been a fortunate man. After all these experiences, you have the moral duty to try to help others. 